\title{
Deconstructing obesity: the face of fatness before and after the discovery of leptin
}

\author{
B. E. Grayson • R. J. Seeley
}

Received: 27 July 2011 / Accepted: 21 September 2011 / Published online: 15 October 2011

(C) Springer-Verlag 2011

\begin{abstract}
The prevalence and severity of obesity have increased to epidemic proportions around the globe, and over two-thirds of the US population grapples with either being overweight or obese. Obesity and its comorbidities not only subtract from quality of overall life, but also claim a substantial cost to life. In this edition of 'Then and now', two seminal papers by D.L. Coleman, 'The influence of genetic background on the expression of the obese $(o b)$ gene in the mouse' and 'Effects of parabiosis of obese with diabetes and normal mice', which featured in Diabetologia in 1973, are appraised for their merit and foresight regarding the present eruption of research into what has consequently been labelled 'the metabolic syndrome'. These two studies determined that a thenunknown circulating factor was responsible for the obese/ diabetic state of the $o b / o b$ mouse by using a parabiosis model. This circulating factor was later dubbed 'leptin'. The present commentary juxtaposes the astute deduction and simple methods used over 35 years ago and modern research methods as we go forth in our effort to successfully treat and prevent obesity, diabetes and their co-morbidities.
\end{abstract}

Keywords $d b / d b \cdot$ Diabetes $\cdot$ Leptin $\cdot o b / o b \cdot$ Obesity . Parabiosis

B. E. Grayson • R. J. Seeley $(\square)$

Metabolic Diseases Institute, University of Cincinnati,

2170 E. Galbraith Road,

Cincinnati, OH 45237, USA

e-mail: randy.seeley@uc.edu
Prior to the $1950 \mathrm{~s}$, it was commonly accepted that the underlying cause of obesity was a lack of willpower and moral strength, the result of gluttony and sloth, and obese individuals were often ridiculed and ostracised. Only a small percentage of the population exhibited obesity, and in the clinic their health problems posed a liability risk for both medicinal treatment and surgery. Although obese individuals are still confronted by harsh sentiments and prejudice, the face of obesity has received a makeover in the last half century. One reason for this is that in the $1950 \mathrm{~s}$ less than $10 \%$ of the US population was obese, in contrast to the overwhelming $68 \%$ of the population that is characterised as overweight or obese today [1]. In addition, the severity of the obesity is also on the rise [2]. Overweight and obesity have become a norm in our society, as exemplified by many service industries being compelled to deal with issues that arise from increasing numbers of larger people navigating an infrastructure made for the lean.

Today obesity is characterised as component of the metabolic syndrome - a compilation of endocrine, cardiovascular and immune symptoms that includes hyperlipidaemia, diabetes and hypertension. Although obesity can necessarily be attributed to an overconsumption of energydense foods and an underutilisation of energy stores, other aspects, which include stress, sleep disturbances, depression, physical inactivity and familial predispositions, impinge on a physiology that may be wired to store energy for a 'rainy day'.

The facelift of obesity began with the discovery of leptin, but we need to go back much further to understand 
the start of the story. In this first edition of the 'Then and now' series, the contribution of two seminal papers by D.L. Coleman get their turn on the scientific catwalk: 'The influence of genetic background on the expression of the obese $(o b)$ gene in the mouse' [3] and 'Effects of parabiosis of obese with diabetes and normal mice' [4]. These papers begin the tale that turned the tide of attributing obesity from moral incompetence to complex physiological and behavioural responses resulting in dysregulation and ultimate disease of many organ systems.

At the time of the publication of his papers in 1973, Douglas L. Coleman was a researcher at the Jackson Laboratory, in Bar Harbor, Maine [5]. In the first manuscript featured here, co-authored with Katharine P. Hummel, he introduced a newly discovered severely diabetic strain of $o b / o b$ mouse bred onto a congenic background [3]. The original obese mutant $\mathrm{C} 57 \mathrm{BL} / 6 \mathrm{~J}-o b$ mouse exhibited a variable, often mildly diabetic, phenotype, which commenced at a range of ages because of the non-inbred lines from which they originated. The new strain of mouse, C57BK/KsJ-ob, had phenotypic characteristics that were more similar to the recently discovered diabetes $(d b / d b)$ mouse, which exhibited not only obesity and hyperphagia but also demonstrated marked hyperglycaemia and islet cell atrophy. What became evident from the two versions of the $o b / o b$ mouse is that the mutation interplayed with the background metabolic state of the animal to produce physiological changes to meet the need for insulin. The juxtaposition of these two strains of obese mice, along with the newly identified $d b / d b$ mouse, gave Coleman his Eureka moment. He presciently postulated that some circulating factor might be responsible for the profound obesigenic and diabetic phenotype in both mutant animals and that restoration of this circulating factor could rescue the severe sequelae in these animals.

What was Coleman's strategy? It was a clever set of experiments that resulted from adroit deductive reasoning, good old-fashioned cross-pollination of research disciplines, and a sprinkling of serendipity. Another group at the Jackson Laboratory that was involved in anaemia research routinely used a parabiosis model, i.e. anastomosis of pairs of mice. Parabiosis requires the surgical union of two mice, in which their respective skin and musculature are sutured to each other, after the adjacent scapulae and femurs are fused to stabilise the union. During postoperative recovery, the vasculature naturally anastomoses, resulting in a common circulatory system that by all measures exhibits a blood exchange of approximately $1-2 \%$ every minute. In these historic studies, Coleman devised a variety of combinations of parabiont unions to directly test his hypotheses.
In the first set of parabiosis experiments performed by Coleman, $d b / d b$ animals were joined to wild-type $(+/+)$ animals (Fig. 1a). Surprisingly, the $+/+$ members of the pairs consistently expired, and careful carcass analysis resulted in the discovery of an absence of food remnants in their guts and undetectable levels of hepatic glycogen. Coleman's conclusions from these initial studies defined the next rounds of parabiont unions. He deduced that the $d b / d b$ animal produced a circulating signal sufficiently robust to produce profound starvation in a normal animal. In a subsequent experiment, he adjoined $o b / o b$ with wild-type mice and observed marked improvements in glycaemic control in combination with decreased energy intake and decelerated weight gain in the $o b / o b$ members of the pairs (Fig. 1b). In contrast, the union of $o b / o b$ and $d b / d b$ animals resulted in severe weight loss in the $o b / o b$ mouse, hypoglycaemia and even death caused by insufficient intake (Fig. 1c). In this union, the $d b / d b$ mouse gained weight as would have been anticipated by its normal growth curve and there were no improvements in metabolic phenotype. Unions between $o b / o b$ pairs and between $+/+$ pairs (Fig. 1d) were included as controls in these studies. Coleman astutely concluded from these experiments that the $d b / d b$ mouse produced a satiety factor that it could not respond to itself. He further concluded that the $o b / o b$ mouse is unable to produce the satiety factor in sufficient quantities to regulate its own body weight and food intake.

Over two decades passed before the elusive factor of Coleman's hypothesis was identified by Jeffrey Friedman at Rockefeller University (New York), through intensive positional cloning experiments that spanned a 9 year duration. Finally, in 1994, the obese phenotype of the $o b / o b$ mouse was pinned on a hormone eventually dubbed 'leptin' [6]. The cloning and subsequent descriptions of the action of leptin and its receptor on food intake and energy balance were seminal to ushering in a new age in the scientific study of obesity and a more enlightened attitude to individuals who have the disorder.

Since the time of these studies 35 years ago, technology has accelerated the scope and depth to which the genome can be probed. We flank genes with microcassettes to knock them out or overexpress their encoded proteins to supraphysiological levels. This can occur in the whole animal as well as in any desired flavour of cells, the experiments being limited only by our imagination and identification of cell-specific promoters. These geneencoded proteins can bleed the colours of the rainbow into spinning-disk confocal microscopes to establish exact in vivo localisation and movement. Expression profiling can track every gene altered by a particular manipulation to the statistical precision of $p<0.001$. Personalised genome 
a

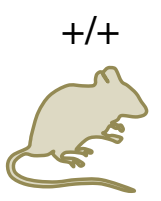

b

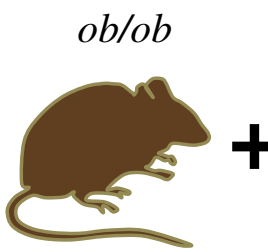

c

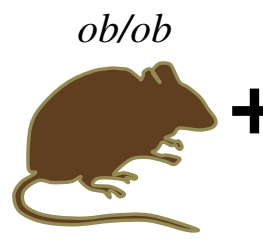

d

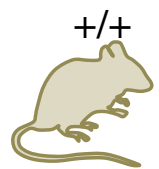

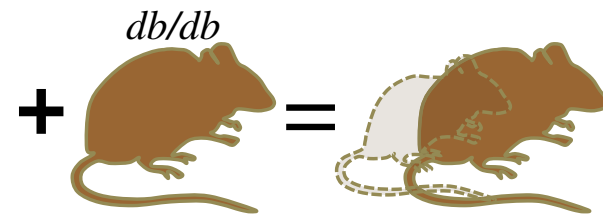

$+/+$

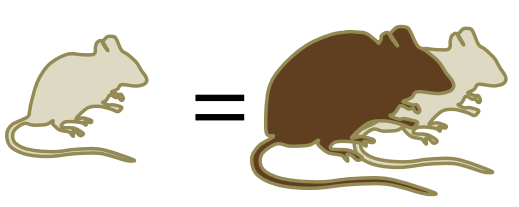

$d b / d b$
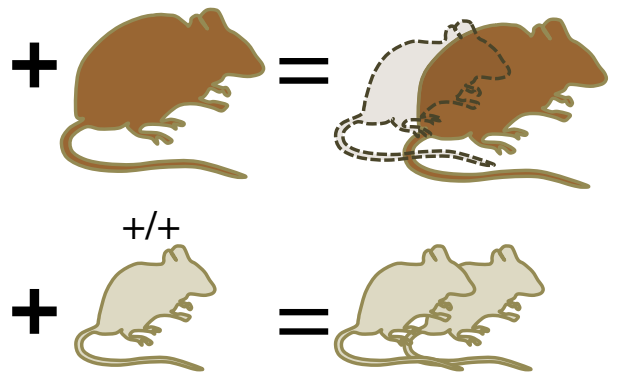

Fig. 1 Summary of parabiosis models. Combinations of mice first reported by Coleman [4]

information is available with complete listings of singlenucleotide polymorphisms that cosegregated over the evolution of our personal family lineage. That said, Coleman's parabiosis technique for testing his hypothesis pales in contrast with flashy modern genetics, i.e. it is an 'old-school' technique, still popular in some fields. Nevertheless, the power of his experimental logic and the clear-cut findings that resulted from them allowed him to directly test and validate his hypotheses. No matter how crude one might think such parabiosis experiments to be relative to recent technological advances, they changed the face of obesity.

There are many unanswered questions that resist our sophisticated efforts to understand the regulation of energy balance and food intake. We have not yet determined how to rescue ourselves from drowning in our own adiposity. And the quest continues for the existence of additional adipokines that might account for some of the gaps in our understanding. Perhaps our current use of genetically modified organisms impedes our ability to identify the experiments that might hold more answers to our dilemma. Coleman's first paper featured here introduced the thennovel $o b / o b$ mouse, which has a phenotype that emerges most robustly on a congenic background. This early manuscript warned of the dangers of weighing the strength of a phenotype against a particular genotype. We often assume the gene of our choice has one overriding function throughout the development of the organism, and we thus fail to account for the pleiotropic roles any gene can exact in the continuity of an organism's existence. An important question is: Do our modern genetic models recapitulate the true mechanism and action of a gene? Indeed, Coleman's insights irrevocably changed obesity research. His findings led directly to the transformation of obesity research, from a small endeavour happening in the shadows of science to a discipline that attracts strong scientists from myriad backgrounds. The most obvious outward sign of this is the presentation of the Albert Lasker Basic Medical Research Award to Coleman and Friedman for their contributions to identifying leptin. This discovery extended our imaginations to include a circulating factor that provides information from adipose tissue to the brain.

Much remains to be done before we go from an understanding of obesity to being successful in reducing its prevalence and its consequences. All of us can take a lesson from Coleman and his strategy. Clear experimental logic can provide monumental insights even when used on what might now be considered crude experimental techniques such as parabiosis. Combining such logic and older techniques with the power of modern tools has the potential 
to change not only the face of obesity but, we hope, the size of its body as well.

Acknowledgements We thank the S.C. Woods, Department of Psychiatry and Behavioral Neuroscience, for careful reading and editing of this manuscript. The work of the laboratory is supported in part by the National Institutes of Health (NIH) Awards DK56863, DK57900, U01CA141464, DK082480, MH069860 and also work with Ethicon Endo-Surgery Inc., F. Hoffman-La Roche Ltd, Pfizer Inc. and Novo Nordisk A/S. B.E. Grayson is also supported by NIH Award 1F32HD68103-01A.

Contribution statement BEG and RJS were responsible for interpretation of data and literature, drafting of the manuscript and approval of the final version for publication.

Duality of interest statement The authors declare that there is no duality of interest associated with this manuscript.

\section{References}

1. Flegal KM, Carroll MD, Ogden CL, Curtin LR (2010) Prevalence and trends in obesity among US adults, 1999-2008. JAMA 303:235-241

2. Centers for Disease Control and Prevention (2010) Vital signs: state-specific obesity prevalence among adults-United States, 2009. MMWR Morb Mortal Wkly Rep 59:951-955

3. Coleman DL, Hummel KP (1973) The influence of genetic background on the expression of the obese $(o b)$ gene in the mouse. Diabetologia 9:287-293

4. Coleman DL (1973) Effects of parabiosis of obese with diabetes and normal mice. Diabetologia 9:294-298

5. Coleman DL (2010) A historical perspective on leptin. Nat Med 16:1097-1099

6. Zhang Y, Proenca R, Maffei M, Barone M, Leopold L, Friedman JM (1994) Positional cloning of the mouse obese gene and its human homologue. Nature 372:425-432 